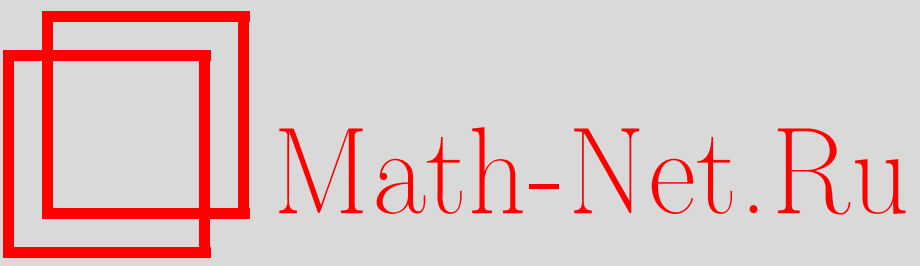

Ф. А. Шамоян, О слабой обратимости в весовых анизотропных пространствах голоморфных в поликруге функций, УМН, 2000, том 55, выпуск 2, 153-154

DOI: https://doi.org/10.4213/rm284

Использование Общероссийского математического портала Math-Net.Ru подразумевает, что вы прочитали и согласны с пользовательским соглашением

http://www.mathnet.ru/rus/agreement

Параметры загрузки:

IP: 54.147 .182 .235

26 апреля 2023 г., 17:39:49 


\title{
О СЛАБОЙ ОБРАТИМОСТИ В ВЕСОВЫХ АНИЗОТРОПНЫХ ПРОСТРАНСТВАХ ГОЛОМОРФНЫХ В ПОЛИКРУГЕ ФУНКЦИЙ
}

\author{
Ф. А. ШАМоян
}

Пусть

$$
U^{n}=\left\{z=\left(z_{1}, \ldots, z_{n}\right),\left|z_{j}\right|<1, j=\overline{1, n}\right\}
$$

- единичный поликруг в $n$-мерном комплексном пространстве $\mathbb{C}^{n}$,

$$
\varphi(r)=\left(\varphi_{1}\left(r_{1}\right), \ldots, \varphi_{n}\left(r_{n}\right)\right), \quad r=\left(r_{1}, \ldots, r_{n}\right) \in \mathbb{R}_{+}^{n},
$$

функции $\varphi_{j}, j=\overline{1, n}$, являются монотонно растущими, положительными функциями на $(0,+\infty)$. Через $A^{p}(\varphi)(p>0)$ обозначим пространство голоморфных в $U^{n}$ функций, для которых

$$
\|f\|_{A^{p}(\varphi)}=\left(\int_{U^{n}}|f(z)|^{p} \exp \left(-\varphi\left(\frac{1}{1-|z|}\right)\right) d m_{2 n}(z)\right)^{1 / p}<+\infty,
$$

где $m_{2 n}-2 n$-мерная мера Лебега на $U^{n}$,

$$
\exp \left(-\varphi\left(\frac{1}{1-|z|}\right)\right)=\prod_{j=1}^{n} \exp \left(-\varphi_{j}\left(\frac{1}{1-\left|z_{j}\right|}\right)\right), \quad z=\left(z_{1}, \ldots, z_{n}\right) \in U^{n} .
$$

В дальнейшем вектор-функцию $\varphi$ с вышеуказанными свойствами назовем весовой. Символом $H^{p}\left(U^{n}\right), 0<p \leqslant+\infty$, обозначим классы Харди в $U^{n}$ (см. [1]).

Пусть $X$ - некоторое топологическое пространство голоморфных функций в $U^{n}$, в котором множество всех многочленов от $z_{1}, \ldots, z_{n}$ всюду плотно, при этом операторы

$\delta_{z}(f)=f(z), \quad T(f)\left(z_{1}, \ldots, z_{n}\right)=z_{1} \cdot z_{2} \cdots z_{n} \cdot f\left(z_{1}, \ldots, z_{n}\right), \quad z=\left(z_{1}, \ldots, z_{n}\right) \in U^{n}$, являются непрерывными в $X$.

ОПредЕлЕниЕ. Пусть $f \in X, f(z) \neq 0, z \in U^{n}$, и существует последовательность многочленов $\left\{P_{n}\right\}_{1}^{\infty}$ такая, что $\lim _{n \rightarrow+\infty} P_{n} f=1$, причем сходимость имеет место в топологии пространства $X$. Тогда говорят, что $f$ слабо обратимый элемент пространства $X$.

Отметим, что слабая обратимость функции $f$ равносильна ее цикличности для оператора сдвига $S(f)\left(z_{1}, \ldots, z_{n}\right)=z_{1} \cdots z_{n} f\left(z_{1}, \ldots, z_{n}\right)$, т.е. полноте системы $\left\{S^{k}(f)\right\}_{k \in \mathbb{Z}_{+}^{n}}$ в пространстве $X$.

В этой заметке мы получим полное описание тех весов $\varphi$, для которых каждая $f \in H^{\infty}\left(U^{n}\right)$, $f(z) \neq 0, z \in U^{n}$, является слабо обратимой в пространстве $A^{p}(\varphi)$.

Теорема 1. Пусть $\varphi=\left(\varphi_{1}, \ldots, \varphi_{n}\right)$ - весовая вектор-функция на $\mathbb{R}_{+}$,

$$
\begin{aligned}
m_{k}^{(j)} & =\sup _{0<r<1}\left(r^{k} \exp \left(-\varphi_{j}\left(\frac{1}{1-r}\right)\right)\right), \\
M_{k}^{(j)} & =\sup _{k \geqslant 1}\left(k^{m} m_{k}^{(j)}\right), \quad m=0,1,2, \ldots, \\
T_{j}(r) & =\sup _{m \geqslant 1}\left(\frac{r^{m}}{M_{m}^{(j)}}\right), \quad r \in(0,+\infty), \quad j=\overline{1, n} .
\end{aligned}
$$

Тогда если

$$
\int^{+\infty} \frac{\lg T_{j}(r)}{r^{3 / 2}} d r=+\infty, \quad j=\overline{1, n},
$$

то каждая $f \in H^{\infty}\left(U^{n}\right), f(z) \neq 0, z \in \underline{U^{n}}$, слабо обратима в $A^{p}(\varphi)(1 \leqslant p<+\infty)$.

Обратно, если функции $\varphi_{j}\left(e^{y}\right), j=\overline{1, n}$, являются выпуклыми и при некотором $j_{0}$, $1 \leqslant j_{0} \leqslant n$, интеграл (4) сходится, то существует функиия $f \in H^{\infty}\left(U^{n}\right), f(z) \neq 0$, $z \in U^{n}$, не слабо обратимая в $A^{p}(\varphi)$ при всех $1 \leqslant p<+\infty$.

Работа частично поддержана МО РФ (грант № 97-0-1.6-110) и РФФИ (грант № 99-01-00103). 
ЗАмЕЧАНИЕ. В одномерном случае при более жестких ограничениях на регулярность роста функции $\varphi$ в других терминах аналогичньй резултат получен Н.К. Никольским в [2], а в пространстве голоморфных в круге функций с заданным ростом коэффицциентов разложения А. Берлингом в [3].

Напомним, что (см. [1]) функция $f \in H^{p}\left(U^{n}\right)$ называется внешней, если

$$
\ln |f(0)|=\frac{1}{(2 \pi)^{n}} \int_{T^{n}} \ln |f(\zeta)| d m_{n}(\zeta),
$$

где $T^{n}$ - остов поликруга $U^{n}, d m_{n}-n$-мерная мера Лебега на $T^{n}$. При $n=1$, как установлено Н.К. Никольским (см. [2]), если $f \in A^{p}(\varphi), 1 \leqslant p<+\infty,-$ внешняя функция, то $f$ слабо обратима в $A^{p}(\varphi)$ при произвольных весах $\varphi$. Очевидно, что в этих пространствах не всякая внутренняя функция слабо обратима (см. [4]-[6]). В многомерном случае справедлива

ТЕОРема 2. Пусть $\varphi=\left(\varphi_{0}, \ldots, \varphi_{0}\right)-$ весовая, при әтом функиия $y \rightarrow \varphi_{0}\left(e^{y}\right)$ выпукла на $(0,+\infty)$. Тогда следующие утверждения равносильны:

1) каждая внешняя функчия $f \in H^{\infty}\left(U^{n}\right)$ слабо обратима в пространстве $A^{p}(\varphi)$, $1 \leqslant p<+\infty$

2) $\int_{1}^{+\infty} \frac{\lg T_{0}(r)}{r^{3 / 2}} d r=+\infty$, әде $T_{0}(r)$ определяется по (3),

3) каждая функция $f \in H^{\infty}\left(U^{n}\right), f(z) \neq 0, z \in U^{n}$, (в частности, каждая внутренняя функиия) слабо обратима в $A^{p}(\varphi)$.

Доказательство теорем 1 и 2 основано на следующих вспомогательных утверждениях.

Лемма 1. Пусть $f \in H^{\infty}\left(U^{n}\right), f(z) \neq 0, z \in U^{n}$,

$$
D_{j} f(z)=z_{j} \frac{\partial f(z)}{\partial z_{j}}, \quad D^{k} f\left(z_{1}, z_{2}, \ldots, z_{n}\right)=D_{n}^{k_{n}} D_{n-1}^{k_{n-1}} \cdots D_{1}^{k_{1}} f\left(z_{1}, \ldots, z_{n}\right),
$$

$z=\left(z_{1}, \ldots, z_{n}\right) \in U^{n}, k=\left(k_{1}, \ldots, k_{n}\right) \in \mathbb{Z}_{+}^{n}$. Тогда существует голоморфная в $U^{n}$ функция $\psi_{k}$ такая, что $D^{k} f(z)=\psi_{k}(z) f(z)$, причем

$$
\left|\psi_{k}\left(z_{1}, \ldots, z_{n}\right)\right| \leqslant \frac{C(k)\|f\|_{\infty}}{\left(1-\left|z_{1}\right|\right)^{3 k_{1}} \cdots\left(1-\left|z_{n}\right|\right)^{3 k_{n}}} .
$$

Лемма 2. Пусть $f \in A^{p}(\varphi), \varphi$ - весовая вектор-функиия. Если $f\left(z_{1}, \ldots, z_{n}\right)=$ $\sum_{|k|=0}^{+\infty} a_{k_{1}, \ldots, k_{n}} z_{1}^{k_{1}} \ldots z_{n}^{k_{n}}, z=\left(z_{1}, \ldots, z_{n}\right) \in U^{n}, k=\left(k_{1}, \ldots, k_{n}\right)$, то справедлива очен$\frac{\left|a_{k_{1}, \ldots, k_{n}}\right|}{\left(1+k_{1}\right) \cdots\left(1+k_{n}\right)} \leqslant \frac{\|f\|_{A^{p}(\varphi)}}{\sup _{r \in \mathbb{Q}^{n}}\left\{r^{k} \exp \left\{\frac{1}{p} \varphi\left(\frac{1}{1-r}\right)\right\}\right\}}, \quad k=\left(k_{1}, \ldots, k_{n}\right), \quad r^{k}=r_{1}^{k_{1}} \cdots r_{n}^{k_{n}}$.

В следующей лемме дается оценка роста функции $f \in A^{p}(\varphi)$ на диагонали поликруга.

Лемма 3. Пусть $f \in A^{p}(\varphi), 1 \leqslant p<+\infty, D f(z)=f(z, z, \ldots, z), z \in U=U^{1}$. Тогда имеет место оценка

$$
\int_{U}|D f(z)|^{p} \exp \left(-\varphi\left(\frac{2}{1-|z|}\right)\right)(1-|z|)^{2 n-2} d m_{2}(z) \leqslant c\|f\|_{A^{p}(\varphi)}^{p} .
$$

\section{СПИСОК ЛИТЕРАТУРЫ}

[1] Рудин У. Теория функций в поликруге. М.: Мир, 1974. [2] Никольский Н. К. Избранные задачи весовой аппроксимации и спектрального анализа // Труды МИАН. 1974. Т. 120. [3] Beurling A. // Acta Math. 1964. V. 112. № 3-4. P. 239-255. [4] Мергелян С. Н. // УМН. 1953. Т. 8. № 4. С. 3-63. [5] Шапиро Г. // Матем. сб. 1967. Т. 73(115). С. 320-330. [6] Шамоян Ф. А. // Изв. АН Арм. ССР. Сер. Матем. 1970. Т. 5. № 5. С. 419-433. 